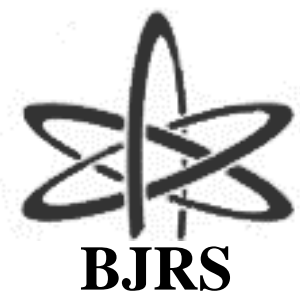

BRAZILIAN JOURNAL

$\mathrm{OF}$

RADIATION SCIENCES

03-1A (2015) 01-07

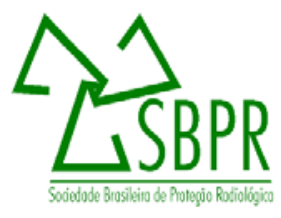

\title{
A importância do uso do dosímetro nos profissionais médicos no serviço de hemodinâmica
}

\author{
Francisca A. de Melo ${ }^{1,2}$, Carla V. da Silva ${ }^{3}$, Décio C. Guerra ${ }^{4}$, Tayline T. dos Santos ${ }^{3}$ e Edgard \\ Victor Filho ${ }^{1}$
}

\author{
${ }^{1}$ Hospital das Clinicas - Universidade Federal de Pernambuco (UFPE) \\ Av. Professor Moraes Rego, $n^{\circ} 1235$ - CDU \\ 50670-901 Recife - PE - Brasil \\ francissamello@yahoo.com.br \\ edgardvf@hotmail.com
}

2Instituto Materno Infantil de Pernambuco (IMIP)

Rua dos Coelhos, $n^{\circ} 300$ - Boa Vista

50070-550 Recife - PE - Brasil

${ }^{3}$ Instituto Federal de Educação,Ciência e Tecnologia de Pernambuco (IFPE)

Av. Professor Luiz Freire, $n^{\circ} 500$ - CDU

50740-540 Recife - PE - Brasil

carla_vieira85@hotmail.com

taylinetyene@hotmail.com

${ }^{4}$ Universidade Federal de Sergipe - (UFS)

Rua Padre Alvares Pitangueira, $n^{\circ} 248$

49400-000 - Lagarto - SE - Brasil

Decio.cavalcanti@yahoo.com.br

\section{RESUMO}

O objetivo deste trabalho é avaliar as exposições médicas às radiações ionizantes do tipo X em um serviço de radiologia intervencionista, de um hospital universitário, fazendo uma correlação com a importância do uso de dosímetros para monitoração da dose efetiva nos Indivíduos Ocupacionalmente Expostos (IOE). Foi realizada a análise das doses efetiva por meio de relatórios de dosímetro tipo filme, emitidos mensalmente por laboratório de proteção radiológica. Foi então, feita a análise das doses de radiação em dois momentos; no primeiro não houve nenhuma orientação quanto à necessidade do uso de dosímetros, no segundo momento os profissionais realizaram todos os procedimentos portando o dosímetro. O resultado mostrou uma dose efetiva média dos profissionais/ano de 8,60 mSv no primeiro momento, contra uma dose de 27,41 mSv no segundo momento após a utilização rotineira dos dosímetros, ultrapassando nesta segunda fase, a taxa de dose anual permitida pela legislação de proteção radiológica vigente, que preconiza $20 \mathrm{mSv}$ /ano por profissional. A comparação resultou em um aumento de dose efetiva dos profissionais em aproximadamente 300\%. Conclui-se que a implementação de um projeto de educação continuada, incluindo a conscientização da importância no uso diário de dosímetro mostra-se como solução para a otimização das doses desses indivíduos ocupacionalmente exposto. 
Palavras-chave: Dosimetria, Hemodinâmica, IOEs.

\section{INTRODUÇÃO}

Os procedimentos intervencionistas envolvem estudos de longa duração, obtendo muitas imagens radiográficas que são visualizadas em tempo real, e consequentemente, eleva a quantidade de radiação X recebida pelo paciente e equipe clínica [1].

Nos últimos anos, tem sido observada uma notável evolução no campo dos procedimentos intervencionistas, levando a aumento na quantidade, e também na maior complexidade dos procedimentos. Os procedimentos terapêuticos por via percutânea através do uso de catéteres veem substituindo algumas modalidades de tratamento cirúrgico, implicando em menor risco de morte, menor tempo de internação e também de recuperação para a maioria dos pacientes tratados [2]. Além dos cuidados com os pacientes, a proteção da equipe clínica também deve ser levada em consideração durante um procedimento de radiologia intervencionista. No Brasil, os limites de dose para trabalhadores com radiação são aqueles determinados pela CNEN e adotados pela Portaria MS-453/98, do Ministério da Saúde [1].

A finalidade do monitoramento individual é garantir que os limites de dose não sejam excedidos. As estimativas de dose efetiva são obtidas por meio de dosímetros utilizados pela equipe médica durante a realização do procedimento, que representa a taxa de dose no corpo inteiro, órgão ou tecido humano. O uso do dosímetro individual por parte dos profissionais de saúde constitui o principal meio de avaliação da eficácia de um programa de controle de dose individual estabelecido no serviço de radiodiagnóstico [3,4]. É primordial que os dosímetros sejam utilizados corretamente, associados aos Equipamentos de Proteção Individual (EPI’s) e coletivo. A Comissão Internacional em Proteção Radiológica (ICRP) estabelece que nenhuma prática deve ser adotada usando radiação ionizante, a menos que seus benefícios sejam superiores aos detrimentos causados por ela. Dessa maneira, as técnicas envolvendo sua utilização devem ser otimizadas para que as 
doses recebidas sejam tão pequenas quanto possível, mas compatíveis com os propósitos dos diagnósticos [5].

Estudos internacionais mostram que a dose recebida pelo médico é mais alta, quando comparada aos demais componentes da equipe, isto se justifica devido este profissional permanecer por períodos mais prolongados próximo ao paciente e a fonte de raios-X [6]. A partir do exposto, este estudo objetiva analisar a dose efetiva recebida pelos profissionais médicos envolvidos na realização de procedimentos de hemodinâmica, de forma a instituir a importância do uso de dosímetro na monitoração individual.

\section{MATERIAIS E MÉTODOS}

Trata-se de um estudo quantitativo, quase experimental tipo antes e depois. A presente pesquisa foi desenvolvida no serviço de hemodinâmica de um hospital universitário na cidade de RecifePE entre os anos de 2012 a 2013. No referido serviço são realizados procedimentos do sistema cardiovascular, entre eles: cateterismo cardíaco, angioplastia, arteriografia cerebral, colangiografia, quimioembolização hepática, entre outros. O serviço realiza aproximadamente 150 procedimentos/mês. Setenta e três por cento desses procedimentos são realizados pela radiologia intervencionista e cirurgia endovascular. $\mathrm{O}$ angiógrafo utilizado nos procedimentos é do tipo digital de fluorografia, modelo DFP 2000 A, com intensificador de imagem. Todas as aquisições foram realizadas no modo pulsado e o equipamento mantém sua excelência na segurança de funcionamento, por meio de manutenções periódicas e levantamentos radiométricos. Para realização do estudo foram considerados como requisitos básicos: o uso dos dosímetros tipo filme, a análise dos dados de monitoração individual dos profissionais médicos obtidas por um laboratório de proteção radiológica e a comparação das doses obtidas com as preconizadas pela CNEN e Portaria 453/98, do Ministério da Saúde.

No período do estudo foi realizada a análise dos relatórios das doses efetiva nos profissionais médicos, e esta avaliação foi dividida em dois momentos, para efeito comparativo. No primeiro não houve nenhuma orientação para a necessidade do uso de dosímetro. No segundo, os 
profissionais só realizavam procedimentos portando o dosímetro. O relatório de doses de radiação é emitido após leitura ópitca dos filmes dosimétricos sobre os diferentes filtros, através do densitômetro. Os detectores foram posicionados sobre o avental plumbífero de 0,5 mm de espessura nos IOE, na região anterior do tórax. Os valores obtidos foram divididos por 10, como determinado pela legislação vigente. O serviço não oferece os equipamentos de proteção coletiva, como o protetor suspenso e o saiote plumbífero. As taxas de dose obtidas foram comparadas a valores estabelecidos pelo princípio de radioproteção “limitação de dose individual”, preconizada pela Portaria 453/98 do Ministério da saúde.

\section{RESULTADOS E DISCUSSÕES}

O relatório de doses de radiação reflete o tempo de exposição ocupacional individual. De acordo com a carga horária do profissional e quantidade de procedimentos realizados, eles irão apresentar uma maior ou menor exposição à radiação. Foram constatadas doses diferentes na mesma categoria profissional, devido à atitude na condução dos exames. As doses registradas na monitoração individual foram tomadas em condições uniformes. Todos os dosímetros foram utilizados sobre o avental plumbífero de $0,5 \mathrm{~mm}$ de espessura.

A Figura 1 mostra os valores das doses efetivas da monitoração individual dos profissionais médicos no período de 2012, sem orientação para o uso de dosímetro.

Figura 1. Tabela de Doses Efetivas sem uso de dosímetro

\begin{tabular}{|c|c|c|c|c|c|c|c|c|c|c|c|c|}
\hline \multicolumn{13}{|c|}{ DOSES MÉDICO - HEMODINÂMICA - 2012} \\
\hline PROFISSIONAIS & JAN & FEV & MAR & ABR & MAI & JUN & JUL & AGO & SET & OUT & NOV & DEZ \\
\hline M1 & 0,2 & 0,2 & 0,2 & 0,2 & 0,2 & 0,2 & 0,2 & 0,2 & 0,2 & 0,29 & 0,8 & 0,2 \\
\hline M2 & 0,2 & 0,2 & 0,2 & 0,2 & 0,22 & 0,2 & 0,2 & 0,2 & 0,43 & 0,2 & 0,2 & 0,23 \\
\hline M3 & 0,2 & 0,2 & 0,2 & 0,2 & 0,2 & 0,2 & 0,2 & 0,2 & 0,2 & 2,25 & 0,2 & 4 \\
\hline M4 & 0,2 & 0,2 & 0,71 & 1,03 & 4,8 & 0,2 & 0,2 & 0,34 & 0,54 & 0,2 & 1,49 & 1,01 \\
\hline M5 & 0,2 & 0,2 & 0,2 & 0,2 & 0,2 & 0,2 & 0,2 & 0,2 & 0,2 & 0,2 & 0,99 & 0,2 \\
\hline M6 & & & & & 0,2 & 0,2 & 0,2 & 0,2 & 0,67 & 3,07 & 1,96 & 0,2 \\
\hline M7 & & & & & 0,2 & 0,2 & 0,2 & 0,2 & 0,2 & 0,48 & 5,62 & 0,2 \\
\hline M8 & & & & & & 0,2 & 0,28 & 0,42 & 0,2 & 0,2 & 0,31 & 0,76 \\
\hline M9 & & & & & & 0,2 & 0,2 & 1,38 & 0,2 & 0,2 & 0,2 & 0,27 \\
\hline M10 & & & & & & & & & 0,56 & 4,25 & 0,38 & 0,2 \\
\hline M11 & & & & & & & & & & 4,4 & 6,61 & 0,66 \\
\hline M12 & & & & & & & & & & 2,45 & 32,24 & 7,37 \\
\hline M13 & & & & & & & & & & & & 0,22 \\
\hline
\end{tabular}


A dose efetiva média dos profissionais/ano no primeiro momento foi de 8,60 mSv.

Os baixos valores de dose no primeiro momento de acordo com a Figura 1 podem ser explicados pela não utilização frequente dos dosímetros, visto que o número de procedimentos permaneceu constante, assim como, o equipamento manteve sua excelência através de manutenções periódicas e levantamentos radiométricos.

A Figura 2 exibe os valores das doses efetiva da monitoração individual dos profissionais médicos em 2013, com orientação para o uso de dosímetro.

Figura 2. Tabela de Doses efetivas com uso obrigatório de dosímetros.

\begin{tabular}{|c|c|c|c|c|c|c|c|c|c|c|c|c|}
\hline \multicolumn{13}{|c|}{ DOSE MÉDICO - HEMODINÂMICA - 2013} \\
\hline PROFISSIONAIS & JAN & FEV & MAR & ABR & MAI & JUN & JUL & AGOS & SET & OUT & NOV & DEZ \\
\hline M1 & & 0,2 & 0,2 & 0,2 & 0,2 & 0,2 & 0,2 & 0,2 & 0,2 & 0,2 & 0,2 & 0,2 \\
\hline M2 & & 0,2 & 0,2 & 0,2 & 0,3 & 0,3 & 0,31 & 0,67 & 0,47 & 1,39 & 0,46 & 0,64 \\
\hline M3 & & 0,88 & 0,2 & 0,2 & 0,97 & 0,97 & 0,2 & 1,51 & 5,32 & 1,01 & 1,35 & 1,57 \\
\hline M4 & & 0,88 & 0,2 & 1,93 & 0,33 & 0,33 & 1,01 & 1,52 & 4,41 & 1,27 & 2,54 & 0,4 \\
\hline M5 & & 0,28 & 0,2 & 0,2 & 0,3 & 0,3 & 0,2 & 0,2 & 0,2 & 0,2 & 0,2 & 1,38 \\
\hline M6 & & 0,2 & 0,2 & 2,34 & 0,2 & 0,2 & 0,2 & 0,2 & 0,2 & 0,2 & 0,2 & 0,2 \\
\hline M7 & & 1,36 & 0,2 & 8,85 & 6,1 & 6,1 & 2,6 & 5,09 & 6,89 & 2,1 & 1,48 & 1,28 \\
\hline M8 & & 1.07 & 0,2 & 0,79 & 0,64 & 0,64 & 0,46 & 6,62 & 1.43 & 0,8 & 0,83 & 0,74 \\
\hline M9 & & 0,2 & 0,2 & 3,72 & 0,4 & 0,4 & 0,2 & 0,2 & 0,2 & 0,63 & 5,05 & 0,2 \\
\hline M10 & & 0,21 & 0,2 & 0,2 & 0,2 & 0,2 & 0,2 & 0,2 & 0,36 & 0,37 & 0,2 & 0,52 \\
\hline M11 & & 2,91 & 0,2 & 0,2 & 2,14 & 2,14 & 7,1 & 9,08 & 10,82 & 1,4 & 3,42 & 3,03 \\
\hline M12 & & 0,2 & 0,2 & 0,2 & 4,97 & 4,97 & 5,61 & 0,2 & 7,82 & 6,05 & 0,2 & 2,7 \\
\hline M13 & & 0,2 & 0,2 & 0,2 & 0,22 & 0,22 & 0,43 & 0,2 & 0,2 & 0,2 & 0,2 & 0,2 \\
\hline M14 & & 9,19 & 0,2 & 0,2 & 0,2 & 0,2 & 0,2 & 0,2 & 0,2 & 0,2 & 0,2 & 0,2 \\
\hline M15 & & 0,2 & 0,2 & 0,48 & 0,4 & 0,4 & 0,75 & 3,09 & 0,2 & 0,2 & 1,27 & 0,2 \\
\hline M16 & & 0,2 & 0,2 & 0,2 & 0,2 & 0,2 & 0,2 & 0,2 & 0,2 & 0,2 & 0,2 & 0,2 \\
\hline M17 & & & & & 12,89 & 12,89 & 3,12 & 38,14 & 24,12 & 49,19 & 13,29 & 16,54 \\
\hline M18 & & & & & 13,04 & 13,04 & 14,04 & 52,56 & 27,34 & 20,62 & 8,75 & 32,47 \\
\hline
\end{tabular}

*A tabela não contabiliza os valores de doses referentes ao mês de janeiro, não implicando no resultado final.

A dose efetiva media no segundo momento foi de $27,41 \mathrm{mSv}$ ultrapassando a taxa de dose permitida anualmente pela legislação de proteção radiológica vigente, que preconiza 20 mSv/ano para profissional, como exibe a Figura 3.

Figura 3. Tabela de dose dos limites anuais estabelecidos pela CNEN de acordo com a Resolução 114/2011.

\section{LIMITES ANUAIS}

\begin{tabular}{cccc}
\hline Grandeza & Órgão & Individuo Ocupacionalmente Exposto & Indivíduo Público \\
\hline Dose Efetiva & Corpo Inteiro & $20 \mathrm{mSv}$ & $1 \mathrm{mSv}$ \\
\hline
\end{tabular}


Quando correlacionado os anos de 2012 a 2013, as doses efetivas resultaram em um aumento de aproximadamente 300\% em relação ao primeiro período, conforme Figura 4.

Figura 4. Gráfico comparativo das doses.

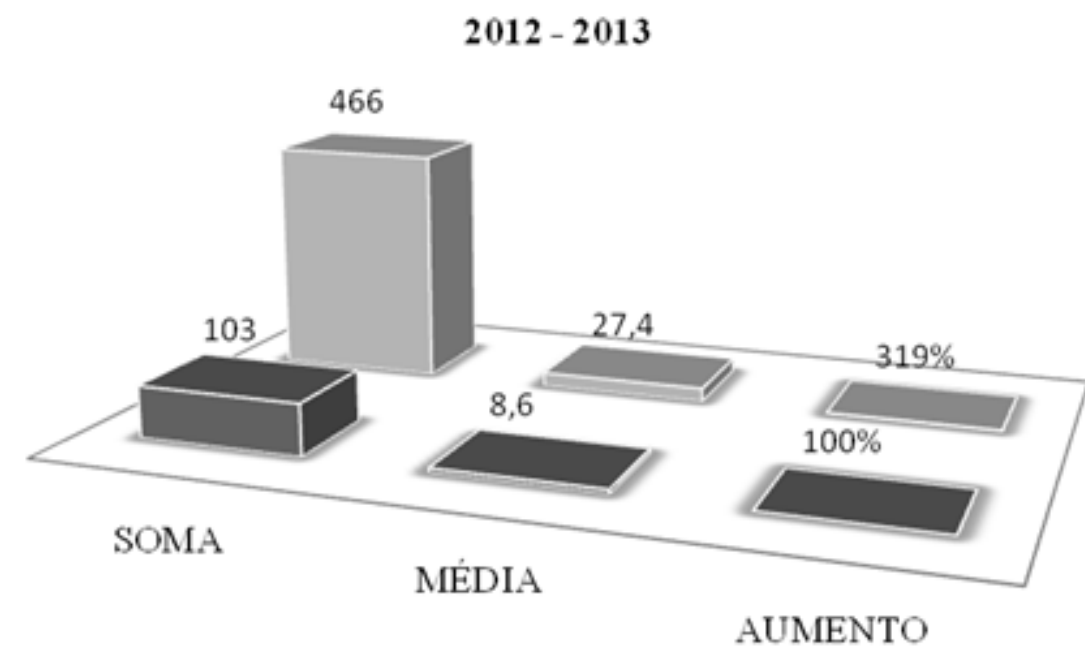

Para efeito comparativo, houve um aumento significativo no uso dos dosímetros e na aplicabilidade dos princípios básicos de radioproteção, quando correlacionado ao primeiro período do estudo.

O estudo também possibilitou averiguar a diferença nas doses entre médicos residentes e seus preceptores, a Figura 5 reflete um aumento significativo nas doses desses profissionais.

Figura 5: Tabela comparativa das doses entre médicos residentes e seu preceptores

\begin{tabular}{|c|c|c|}
\hline \multicolumn{3}{|c|}{ DOSES HEMODINÂMICA MÉDICOS } \\
\hline & RESIDENTES & PRECEPTORES \\
\hline 2012 & 0,57 & 0,22 \\
\hline 2013 & 3,81 & 0,59 \\
\hline SOMA & 4,38 & 0,81 \\
\hline MÉDIA & 2,19 & 0,41 \\
\hline AUMENTO & $541 \%$ & $100 \%$ \\
\hline
\end{tabular}


*A dose média foi divida por 10, devido os dosímetros ser utilizado sobre o EPI.

A Figura 5 é justificável devido os médicos residentes permanecerem por mais tempo na realização dos procedimentos e pela falta de habilidade no manuseio das técnicas.

\section{REFERÊNCIAS}

1. Carnelave F. C., Radiologia Intervencionista e Cirurgia Endovascular, R.J. (2006).

2. Faulkner K. Radiation protection in interventional radiology. Br.(1997).

3. Silva, A. J, Avaliação da dose ocupacional oriunda dos procedimentos especiais guiados por fluoroscopia: cateterismo cardiaco, S.P, Segunda Edição. (2011).

4. Silva, C. eat al, Avaliação da exposição ocupacional em procedimentos de hemodinâmica, S.P, pp 123-126. (2006).

5. International Commission on Radiological Protection. Conversion coefficients for use in radiological protection against external radiation. ICRP Publication 74. New York: Pergamon Press. 1997.

6. . Gronchi C. C., eat al; Controle da exposição ocupacional às radiações ionizantes nos serviços de hemodinâmica segundo portaria 453 e americam college of cardiology, S.P, Volume 2 , pp.2-5. (2009) 